\title{
A importância e a história dos estudos de utilização de medicamentos
}

\author{
Daniela Oliveira de Melo', Eliane Ribeiro², Sílvia Storpirtis ${ }^{1}$ \\ ${ }^{1}$ Faculdade de Ciências Farmacêuticas, Universidade de São Paulo, ${ }^{2}$ Hospital Universitário, \\ Universidade de São Paulo
}

*Correspondência:

D. O. de Melo

Rua Dr Nogueira Martins, 634 - apto 112

04143-020 - São Paulo - SP, Brasil

E-mail: elianer@hu.usp.br
No mundo atual, onde há ampla necessidade de racionalização de recursos, sobretudo nos países mais pobres, esses estudos apresentam-se como alternativa que permite reduzir custos sem perda de qualidade nos tratamentos médicos, além de terem como função detectar possiveis abusos no uso dos medicamentos ou a ocorrência de eventos adversos. Os medicamentos representam boa parcela dos gastos públicos com saúde e não são substâncias inócuas. Essas são as duas principais razões pelas quais, cada vez mais, se reconhece a necessidade e a importância dos estudos que analisam os tratamentos medicamentosos, em especial nos hospitais, e os dados relativos ao consumo em si. Os últimos possibilitam a aplicação da farmacoeconomia e da farmacoepidemiologia como ferramentas no combate à utilização inadequada de medicamentos e a gastos desnecessários. A pesquisa tornou possível confirmar a importância da ocorrência desses estudos, especialmente em hospitais, visando à redução do gasto com medicamentos. A detecção de desvios, ineficácia e eventos adversos com a utilização inadequada de medicamentos possibilita, em nivel macro, o desenvolvimento de políticas governamentais e, em nível micro, a realização de intervenções educativas - ambas as medidas tendo como objetivo a utilização dos medicamentos de forma racional.
Unitermos

- Farmacoepidemiologia

- Racionalização de recursos

- Economia da saúde

\section{INTRODUÇÃO}

No século XX, com o aumento da eficiência da prevenção de doenças e atendimento médico, a expectativa de vida, menor que quarenta anos até a década de 1940, ultrapassou os sessenta e cinco anos (década de 1990) (WHO, 1997). Todavia, com o crescimento dos custos com equipamentos, materiais e medicamentos, surgiu a impossibilidade do acesso universal aos recursos e tecnologia moderna disponíveis (Crozara, 2001).
Assim, visto que os medicamentos se apresentam como um dos fatores responsáveis pelos gastos com saúde, a questão de sua utilização de modo adequado nunca esteve tão presente no cotidiano da população, nem foi tão discutida: segundo Nascimento (2003), o número de reportagens publicadas em jornais e revistas da grande imprensa escrita sobre medicamentos e saúde durante os anos 70, 80 e 90 foram 26, 135 e 250, respectivamente. É de conhecimento geral a necessidade de formulação e aplicação de política de medicamentos que favoreça a 
obtenção de medicamentos essenciais e promova seu uso racional.

\section{A prescrição e os problemas relacionados à falta de informação confiável sobre medicamentos}

"No começo do século XIX a maioria dos medicamentos era remédios de origem natural, de estrutura química e natureza desconhecidas"(Laporte, Tognoni, Rosenfeld, 1989). Após 1940, ocorreu a introdução maciça de novos fármacos, que trouxeram à população possibilidade de cura para enfermidades até então fatais, sobretudo no campo de doenças infecciosas. Os avanços nas pesquisas de novos fármacos, em conjunto com sua promoção comercial, criaram uma excessiva crença da sociedade em relação ao poder dos medicamentos. Segundo Nascimento (2002), a produção de medicamentos em escala industrial, segundo especificações técnicas e legais, fez com que esses produtos alcançassem papel central na terapêutica, deixando de ser considerado como mero recurso terapêutico. Sua prescrição torna-se quase obrigatória nas consultas médicas, sendo o médico avaliado pelo paciente por meio do número de formas farmacêuticas que prescreve. Assim, a prescrição do medicamento tornou-se sinônimo de boa prática médica, justificando sua enorme demanda. Segundo Osler, "o desejo de tomar o medicamento talvez represente o maior aspecto de distinção entre o homem e os animais" (Castro, 2000).

Como exemplos de motivações que contribuem para a utilização irracional dos medicamentos tem-se a enorme oferta (em quantidade e variedade), a atração por novidades terapêuticas - muitas das quais são apenas variações de fórmulas já conhecidas -, o poderoso marketing e "o direito, supostamente inalienável, do médico em prescrever" (Castro, 2000). O medicamento tem, intrinsecamente, um valor simbólico do desejo e da capacidade de modificar o curso "natural" da doença que está sendo tratada. O fato de prescrevê-lo passou a ser o resultado final de um processo de diagnóstico e decisão, em que os fármacos são o resumo da atitude e das esperanças do médico em relação ao curso de uma doença (Laporte, Tognoni, Rosenfeld, 1989). Sobretudo, a prescrição é um compromisso entre as estruturas sanitárias e seus usuários. $\mathrm{O}$ fato de o medicamento ter se tornado uma ferramenta tão familiar aos médicos aumenta o risco de sua utilização irracional (Laporte, Tognoni, Rosenfeld, 1989).

"Na prescrição reflete-se a disponibilidade de fármacos, a informação que foi distribuída sobre eles, que chegou ao médico e que ele interpreta, assim como as condições - tempo, meios diagnósticos, patologias prevalentes, nas quais se desenvolve a atenção médica" (Laporte, Tognoni, Rosenfeld, 1989). Desse conhecimento sobre o efeito das informações sobre o medicamento na hora da prescrição, nasce a preocupação com sua quantidade e qualidade.

Há grande necessidade de informação objetiva e imparcial sobre os medicamentos - muito distante daquela disseminada pela indústria farmacêutica, praticamente única fonte, tanto para os profissionais como para os consumidores. Essa "falta de informação" sobre o assunto e a promoção farmacêutica distorcida e desenfreada leva a vários problemas, dentre os quais tem-se: escolha inadequada de medicamentos, exposições indevidas a reações adversas que podem ser fatais, aumento da resistência bacteriana, aumento da automedicação - assim como de seus riscos, desperdício de dinheiro por parte do indivíduo e da instituição com medicamentos inúteis e desnecessários (Castro, 2000).

Um fato que se torna comum é a necessidade de informação, sobre o tratamento e medicamentos, por parte dos pacientes e familiares. Tal informação deve provir de fontes fidedignas e atualizadas, o que, muitas vezes, não é possível, dependendo-se apenas dos bulários e da propaganda. Sendo assim, são necessários estudos de utilização dos medicamentos para detectar reações adversas, ineficácia do tratamento, efeitos colaterais, bem como a má utilização dos mesmos, o que possibilitaria a realização de intervenções adequadas e oportunas. Esse problema demonstra ser ainda mais grave nos países em desenvolvimento, onde os compêndios elaborados pela indústria tendem a exagerar os apelos clínicos e suavizar os dados sobre possíveis reações graves ou fatais e a produção de estudos sobre a utilização dos medicamentos é pouco expressiva (Laporte, Tognoni, Rosenfeld, 1989; Bermudez, Bonfim, 1999; Castro, 2000; Sobravime, 2001).

Diante desse contexto, surgiram os Centros de Informação de Medicamentos (CIMs), que têm como meta principal a promoção do uso racional de medicamentos. Esse objetivo, para ser alcançado, deve ser trabalhado em conjunto com a Comissão de Farmácia e Terapêutica (CFT), importante fórum de discussão e divulgação de informações para os profissionais de saúde (Laporte, Tognoni, Rosenfeld, 1989; Castro, 2000; Vidoti et al., 2000).

\section{Atenção aos medicamentos e sua utilização}

Os efeitos benéficos potenciais dos fármacos, em geral, são conhecidos durante sua pesquisa e comercialização. Porém, mesmo na época da introdução dos primeiros antibióticos, a possibilidade das reações adver- 
sas já era conhecida. Segundo Paracelsus (1493-1541), "todas as substâncias são venenos, não há uma que não seja veneno. A posologia correta diferencia o veneno do remédio" (Klaassen, 1985). Os dois episódios mais conhecidos de reações adversas, que inclusive fomentaram a conscientização da necessidade de definir, quantificar, estudar e prevenir os efeitos indesejáveis provocados por medicamentos - foram o emprego de dietilenoglicol como solvente de um xarope de sulfanilamida - que ocorreu nos anos trinta e provocou mais de cem mortes - e o caso da talidomida - seu uso durante a gravidez causou um surto de focomegalia, malformação congênita rara, com cerca de 4000 ocorrências, contabilizando 498 mortes. Especificamente para a talidomida, uma revisão dos trabalhos experimentais, anteriores à sua comercialização, "revelou que foram publicados e mal interpretados dados toxicológicos insuficientes e errôneos"(Lenz, 1980). Com os crescentes custos econômicos dos medicamentos nos sistemas de previdência social, entre os anos 1950 e 1960, a questão da utilização dos medicamentos passou a chamar a atenção das autoridades sanitárias (Laporte, Tognoni, Rosenfeld, 1989). A partir desse quadro, há uma evolução da farmacologia clínica - particularmente da farmacovigilância. Desde os anos 1950 há o emprego do ensaio clínico controlado (ECC) como padrão no processo de avaliação de um medicamento. A principal limitação do ECC é sua restrição a indivíduos ou grupos de pacientes, os quais podem não ser representativos dos futuros usuários e acabam recebendo o tratamento em condições diferentes (Castro, 2000).

Em 1988, a Organização Mundial de Saúde publicou uma visão do uso de medicamentos dividindo o planeta em dois blocos, de acordo com as características do atendimento à população - países desenvolvidos e países em desenvolvimento (Crozara, 2001). Nos países em desenvolvimento, havia pouca ou nenhuma organização quanto ao uso de medicamentos e a políticas que garantissem a disponibilidade dos mesmos (Crozara, 2001). Um terço da população mundial não tem acesso aos medicamentos dito essenciais, enquanto a população dos países ricos consome cerca de $80 \%$ dos medicamentos produzidos no mundo (WHO, 1988)

O Brasil e outros países em desenvolvimento, a partir da década de 1970, preocupados com a falta de medicamentos para a população de menor poder aquisitivo, tentaram desenvolver programas governamentais que garantissem a disponibilidade ao menos dos medicamentos mais importantes. No Brasil, em 1970, foi instituída a Central de Medicamentos (CEME). Apesar de muitos dos programas não terem sucesso, os esforços para garantir os medicamentos essenciais à população geraram o surgimento da
RENAME (Relação Nacional de Medicamentos Essenciais) e dos formulários ou guias terapêuticos - que padronizam os medicamentos utilizados nos hospitais, diminuindo custos e possibilitando melhor atendimento (Crozara, 2001).

No mercado farmacêutico encontram-se substâncias não essenciais, desnecessárias e até perigosas. A promoção comercial amplia, indiscriminadamente, o consumo de medicamentos. Esses são alguns dos fatores que tornam a reforma no campo dos medicamentos urgente e necessária (Laporte, Tognoni, Rosenfeld, 1989; Bermudez, Bonfim, 1999; Sobravime, 2001).

No Brasil, pelo menos $35 \%$ dos medicamentos são adquiridos por automedicação, ou seja, para cada dois medicamentos prescritos, pelo menos um é consumido sem orientação médica, sendo comum a reutilização de receitas (Barros, 1995). Segundo Arrais e colaboradores (1997), em estudo sobre o perfil da automedicação no Brasil, revelou-se a má qualidade da escolha dos medicamentos em termos do valor intrínseco, o que reflete "plenamente o mercado farmacêutico brasileiro, caracterizado pela predominância de produtos desnecessários, intensamente propagandeados para o público em geral”.

\section{Explosão Farmacológica}

Entre os anos 1950 e 1960 ocorreu a chamada "explosão farmacológica" devido aos desenvolvimentos fundamentais em ciências biológicas - possibilitando "melhor compreensão dos mecanismos moleculares, celulares e homeostáticos relacionados com a saúde e a doença" (Laporte, Tognoni, Rosenfeld, 1989) - e às conquistas tecnológicas e econômicas após a Segunda Guerra Mundial.

Entre 1987 e 1988, a indústria farmacêutica apresentou crescimento de $13 \%$, superando o crescimento médio da economia mundial que, em geral, resumiu-se a $4 \%$. Apesar desse fato, de acordo com avaliação realizada pela Administração de Alimentos e Medicamentos dos Estados Unidos (Food and Drug Administration - FDA), relativa a 348 novos medicamentos das 25 maiores corporações farmacêuticas americanas - comercializados no período entre 1981 e 1988 - apenas 3\% (12 medicamentos) foram considerados como importante contribuição com respeito aos tratamentos existentes. Há alguns anos, o Departamento de Saúde da Inglaterra declarou que a pesquisa na área farmacêutica "estava sendo dirigida para lucros comerciais ao invés de atender a uma necessidade terapêutica" (Bermudez, Bonfim, 1999).

Segundo Lunde (1980), não se demonstrou que um número elevado de fármacos resulte em maiores benefícios para a saúde pública em relação a um número mais limitado 
de produtos. Pelo contrário, a existência de número elevado pode dar lugar a confusão em todos os níveis da cadeia do medicamento e constituir desperdício de recursos humanos e de dinheiro (Laporte, Tognoni, Rosenfeld, 1989).

\section{Países em desenvolvimento}

"O medicamento, como arma terapêutica, apresenta dois gumes. Por um lado, seu emprego inadequado pode provocar doenças iatrogênicas. Por outro, enquanto os países desenvolvidos investem na descoberta de novas substâncias com melhores características intrínsecas para o combate de problemas já resolvidos, os países em desenvolvimento ainda apresentam problemas de saúde que emergem das péssimas condições de vida da população, diminuindo a efetividade dos tratamentos medicamentosos já existentes"(Pepe, 2000). Esse quadro de doenças advindo das más condições de vida, nos países mais pobres, desvia as ações de saúde produzindo uma medicina mais curativa que preventiva, intensificando a utilização de medicamentos. Fica evidente diante dessa constatação, a necessidade de conscientização sobre o fato de que a promoção de saúde depende, entre outros fatores, de boas condições sanitárias, boa moradia, alimentação adequada, água potável, não estando resumida à ação farmacológica dos medicamentos (Laporte, Tognoni, Rosenfeld, 1989; Castro, 2000; Pepe, Castro, 2000; Lieber, 2000; Pereira, Penteado, Marcelo, 2000).

A profunda desigualdade social manifesta-se mais uma vez observando-se o contraste entre uma grande parte da população, com limitado acesso a medicamentos devido ao custo e problemas na distribuição, e uma pequena parcela de privilegiados, responsável pela maior parte do consumo de medicamentos no país. Esse quadro pode ser observado a partir dos dados da Tabela I (Brasil, 1999).

"O medicamento é produto de tecnologia cara e por vezes inacessível. Países pobres encontram-se dependentes da importação de fármacos ou matérias-primas para sua fabricação. No Terceiro Mundo, a maior fatia do mercado farmacêutico está em mãos de empre- sas privadas" (Castro, 2000), o que encarece os medicamentos. Os programas relacionados à saúde são diretamente afetados pelas crises econômicas e, como conseqüência principal, tem-se a limitação dos recursos destinados ao financiamento da aquisição de medicamentos. O resultado da associação destes problemas pode ser exemplificado pela diferença na proporção do gasto com saúde pelos governos do Brasil e Estados Unidos: no Brasil, em 2003, do total gasto pelo governo, 10,3\% foram dirigidos à saúde, enquanto nos Estados Unidos esse valor alcançou 18,5\% (WHO, 2006).

Nesses países, diante da situação apresentada, a saída para essa gama de problemas pode ser a racionalização dos recursos existentes e a busca de fontes suplementares de financiamento que garantam a extensão da cobertura a toda população. Considerando-se que o consumo de medicamentos representa parte substancial dos gastos com saúde torna-se evidente a importância de sua utilização racional (Klaassen, 1985; Laporte, Tognoni, Rosenfeld, 1989; Luiza, Castro, Nunes, 1999; Castro, 2000; Lieber, 2000).

\section{A publicidade e os medicamentos}

A promoção comercial é um dos fatores que, atualmente, influencia muito a prescrição de medicamentos e seu consumo, devido à existência de várias opções farmacêuticas para um mesmo fim, torna-se elemento essencial para diferenciação entre os produtos. Os gastos da indústria farmacêutica com publicidade refletem sua importância para o setor. Estudos realizados desde a década de 1970 já relatavam o gigantesco esforço de produtores de medicamentos para influir sobre os hábitos de prescrição, dispensação e uso dos medicamentos. Fica ainda mais evidente a importância da preocupação com a publicidade ao verificar que os pacientes se automedicam utilizando informações de prescrições anteriores quando acreditam que apresentem os sintomas semelhantes aos que geraram a prescrição $(40,0 \%)$, no Brasil - se o médico confiar somente na propaganda para prescrever é possível que o erro da prescrição persista. $\mathrm{O}$ investimento em publicidade chega a ser o dobro do que a indústria gasta em pesquisa - representan-

TABELA I - Renda e participação no mercado e despesa média anual com medicamentos. Brasil - 1998

\begin{tabular}{lccc}
\hline Renda & $\begin{array}{c}\text { População } \\
(\%)\end{array}$ & $\begin{array}{c}\text { Participação no mercado } \\
\text { de medicamentos(\%) }\end{array}$ & $\begin{array}{c}\text { Despesa média } \\
\text { anual (US\$) }\end{array}$ \\
\hline Maior 10 SM & 15 & 48 & 193,40 \\
$4-$ 10 SM & 34 & 36 & 64,15 \\
$0-$ 4 SM & 51 & 16 & 18,95 \\
\hline
\end{tabular}

Fonte: BRASIL, 1999 (SM = salário mínimo). 
do entre $20 \%$ e $30 \%$ das vendas nos países em desenvolvimento. Desta quantidade, destinam-se, via de regra, $60 \%$ a visita médica, $12 \%$ a distribuição de amostras, $7 \%$ aos anúncios enviados pelo correio, $8 \%$ aos anúncios em revistas, $8 \%$ aos congressos e reuniões e $5 \%$ a documentação e material bibliográfico(Laporte, Tognoni, Rosenfeld, 1989). Além disso, é difundida a prática de oferecer gratificações ao balconista, o que acarreta a chamada "empurroterapia" (Arrais et al. 1997; Bermudez, Bonfim, 1999; Castro, 2000). $\mathrm{O}$ interesse das indústrias farmacêuticas pela farmácia justifica-se por ela ser uma intermediária e repassadora de produtos farmacêuticos aos diversos consumidores, já que funciona, muitas vezes, como substituta do médico(Mota, Marques, Fernandes, 2000).

No Brasil, segundo um trabalho dedicado à análise das propagandas sobre medicamentos, entre agosto de 1996 e novembro de 1997, a irregularidade das mesmas chega a ser surpreendente. Na análise de 2.920 propagandas, de 41 revistas, o número de propagandas irregulares somou $2.915(99,83 \%)$ - entre as que se referiam aos medicamentos de venda livre e sob prescrição. A classificação em regular e irregular foi realizada em relação ao Decreto 2018/96 (Brasil, 1996). Pelo estudo observou-se, também, que $48,91 \%$ das propagandas, presentes em tais revistas, referiam-se a produtos que deveriam ser anunciados somente para médicos ou cirurgiões dentistas - contribuindo para que a prescrição desses produtos fosse realizada por pessoas não habilitadas (Santi, 1999).

Em outro estudo brasileiro, a análise de 100 peças publicitárias, entre outubro de 2002 e julho de 2003 , revelou que $100 \%$ dos anúncios desconsideraram um ou mais artigos da Resolução 102/2003, com média de 4,3 irregularidades por anúncio. Em 1994, das 100 peças publicitárias, descumpriu-se o artigo que obriga que conste de forma clara e precisa a contra-indicação principal do medicamentos (Art. $3^{\circ}$, Alínea I) e, em 52 vezes,o artigo que veda a sugestão ou estimulação de diagnósticos, aconselhando tratamento correspondente (Art. 10 ${ }^{\circ}$, Alínea IV) (Nascimento, 2005).

Pode-se afirmar que até o surgimento do Decreto 2018/96 (Brasil, 1996), a legislação brasileira era defasada em relação às regras que regem a propaganda dos medicamentos - a única referência a esse respeito era a Lei 6360/76 (Brasil, 1976), que exigia autorização prévia do Ministério da Saúde para a divulgação da publicidade e restringia a propaganda de medicamentos para os quais a dispensação só é realizada mediante prescrição aos profissionais de saúde. Na Tabela II, segue resumo das principais mudanças entre a legislação de 1976 e 1996.

Na legislação de 1996, os principais tópicos destacados foram: na publicidade deve ser veiculado o registro do produto, referências claras quanto à composição deste, suas finalidades, modo de usar; não devem ser apregoadas propriedades terapêuticas não comprovadas, assim como informações que não sejam passíveis de comprovação científica; torna-se obrigatória a declaração das contra-indicações, indicações, cuidados e advertências sobre o uso do produto; toda propaganda conterá, obrigatoriamente, a frase que indique que ao persistirem os sintomas, o médico deve ser consultado. Também foram adicionadas referências a sanções e penalidades.

A Lei 10167/2000 (Brasil, 2000) altera o Decreto 2018/ 96 (Brasil, 1996) nos quesitos: cartazes, painéis ou pôsteres só poderão ser expostos na parte interna dos locais de venda; não se deve associar o uso do produto à prática de atividades esportistas, nem sugerir ou induzir seu consumo em locais ou situações perigosas, abusivas ou ilegais e não se deve incluir a participação de crianças ou adolescentes. A Resolução 102/2003 (Brasil, 2003) classifica os medicamentos em três categorias e regulamenta detalhadamente a propaganda a ser aplicada a cada uma destas. Sua elaboração foi motivada por um programa de avaliação das propagandas de medicamentos realizado pela ANVISA (Agência Nacional de Vigilância Sanitária) e quatorze faculdades afiliadas ao projeto. Apesar disso, segundo Nascimento (2005), a grande novidade da resolução foi a inserção obrigatória da frase "AO PERSISTIREM OS SINTOMAS, O MÉDICO DEVERÁ SER CONSULTADO", após a propaganda de medicamentos de venda livre.

\section{O balanço financeiro da utilização irracional dos medicamentos}

“Toda 'reforma' tem sido motivada por questões econômicas"(Bermudez, Bonfim, 1999) e, no que se refe-

TABELA II - Mudanças na legislação que rege a publicidade, de 1976 para 1996

\begin{tabular}{lcc}
\hline Itens avaliados & $\mathbf{1 9 7 6}$ & $\mathbf{1 9 9 6}$ \\
\hline Autorização prévia para a vinculação da propaganda & Sim & Não \\
Propaganda de medicamentos controlados destinada exclusivamente aos profissionais de saúde & Sim & Sim \\
Detalhamento sobre o procedimento a ser observado na elaboração e vinculação da propaganda & Não & Sim \\
\hline
\end{tabular}

Fonte: Brasil, 1976, 1996. 
re aos medicamentos, a frase se adapta perfeitamente. Em 2003, o mercado brasileiro movimentou cerca de 5 bilhões de dólares, situando-se entre os 15 países de maior faturamento no varejo (FEBRAFARMA, 2004). Um estudo do Instituto IMS Health, empresa dedicada ao acompanhamento do mercado farmacêutico, estimou crescimento de $5 \%$ ao ano das vendas no Brasil, entre 2001 e 2005 - crescimento baseado tanto na melhoria do poder de compra quanto pela contínua oferta de fármacos novos (Nelson, 2005).

Os medicamentos, ao lado dos recursos de diagnóstico e terapêutica, são a face mais visível da relação entre a saúde das pessoas e o Estado e representam parte importante do custo dos serviços de saúde. A representação dos medicamentos no custo sanitário total dos países em desenvolvimento foi calculada entre $25 \%$ e $66 \%$ (Bermudez, Bonfim, 1999).

"O gasto do Sistema Único de Saúde - SUS com medicamentos é estimado em $\mathrm{R} \$ 2$ bilhões anuais, o equivalente a $20 \%$ de todo o mercado farmacêutico brasileiro" (Remédios, 1999). Entre as medidas básicas para a sobrevivência das instituições de saúde tem-se a racionalização da terapêutica. Desperta atenção o fato de que os gastos com prescrição têm crescido $12 \%$ ao ano, mais que o dobro do aumento de $5 \%$ dos gastos nacionais em saúde (Hensley, 1999; Santel, 2000). A prescrição irracional conduz, em geral, a gastos maiores devido à utilização de medicamentos desnecessários ou inapropriados, como produtos demasiadamente caros ou períodos prolongados de tratamento. "Os dados de um estudo em Gana, indicaram que o gasto farmacêutico nos centros de assistência sanitária poderia ter sido reduzido em $70 \%$ se houvessem seguido as recomendações para tratamentos" (Bermudez, Bonfim, 1999).

"Um problema sério é o que resulta da aplicação de políticas de reforma do setor saúde que descarregam nos usuários porção considerável do gasto com medicamentos"(SOBRAVIME, 2001). Esse fato torna-se ainda mais visível ao compararmos os países ricos, nos quais $70 \%$ das formulações farmacêuticas têm financiamento público, aos países pobres, onde os próprios pacientes pagam entre $50 \%$ e $90 \%$ do preço dos medicamentos (Castro, 2000; SOBRAVIME, 2001).

\section{Metodologia dos estudos de utilização de medicamentos}

De acordo com a Organização Mundial da Saúde, os estudos de utilização de medicamentos atendem importantes fins, dependendo da metodologia empregada, como: descrição de padrões de uso de medicamentos; constatação de variações nos perfis terapêuticos no curso do tempo; avaliação dos efeitos de medidas educativas, informativas, reguladoras; estimativa do número de indivíduos expostos a medicamentos; detecção de doses excessivas, mal uso, doses insuficientes e abuso dos medicamentos; estimativa das necessidades de medicamentos de uma sociedade, entre outros (Crozara, 2001).

Para elaborar este tipo de estudo foi necessário criar métodos aplicáveis internacionalmente. O "Norwegian Medicinal Depot" (NMD) desenvolveu um sistema de classificação de medicamentos conhecido como "Anatomical Therapeutic Chemical" (ATC) e uma unidade de medida uniformizada para possibilitar a comparação estatística dos dados e interpretação mais ampla dos estudos - Definided Daily Dose ou Dose Diária Definida (DDD) (Castro, 2000; Crozara, 2001).

A DDD foi "adotada pelo Drug Utilization Research Group (DURG) e recomendada pela OMS, a partir de 1981, para uso em estudos de utilização de medicamentos" (Brasil, 1996). Essa unidade difere para cada fármaco e representa a "dose média diária suposta do fármaco quando utilizado para sua principal indicação" (Castro, 2000).

A principal vantagem do emprego da DDD é a possibilidade de se fazer comparações entre países ou através do tempo, sem que os resultados sejam comprometidos por mudanças de preço ou de apresentação. Talvez o maior inconveniente de sua utilização seja o fato de que essa medida nem sempre equivale, necessariamente, à dose média prescrita, ou mesmo à dose média ingerida. Faz-se importante frisar que a DDD não se trata de uma dose recomendada, mas de uma unidade de medida que permite comparação entre resultados (Castro, 2000).

Para estudos comparativos em unidades hospitalares foi desenvolvida, em 1980, a DDD/100 leitos-dia -

Cálculo da quantidade de medicamento utilizada, em DDD:

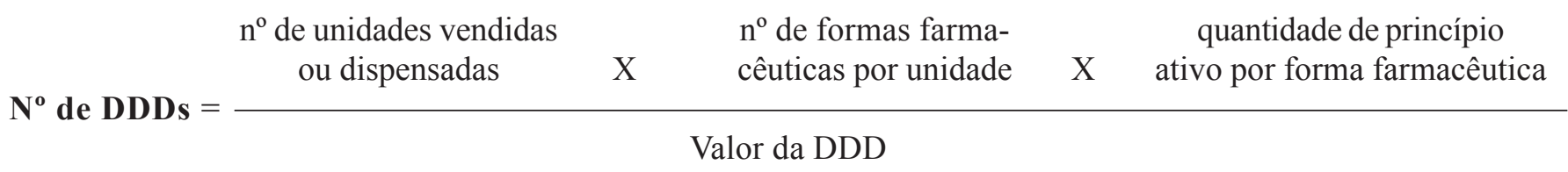


Capellà (1993) e Ruiz (1993) recomendam o cálculo da forma a seguir:

\begin{tabular}{|c|c|c|c|}
\hline & $\mathrm{n}^{\mathrm{o}}$ de DDDs & $X$ & 100 \\
\hline
\end{tabular}

sendo o termo leito-dia referente a "unidade de medida que representa a disponibilidade de leito hospitalar, em um dia" (Remédios, 1999). Hoje, utiliza-se a DDD/100 pacientesdia, pois com essa unidade tem-se estimativa do consumo percentual de dado medicamento em determinado período bem como sugestão da probabilidade de uso de determinado fármaco por um paciente. A interpretação dos termos paciente-dia e leitos-dia gera alguma confusão, porém são termos distintos, assim, leito-dia é cama à disposição do doente durante um dia, e paciente-dia é doente ocupando leito durante um dia (Crozara, 2001).

"Os estudos de utilização incluem aqueles que podem ser realizados dentro do seu conceito, entre os quais mencionam-se: estudo de oferta de medicamentos; estudos quantitativos de consumo de medicamentos; estudos qualitativos sobre a qualidade da prescrição; estudos sobre hábitos de prescrição médica; estudos de cumprimento da prescrição médica; vigilância orientada a problemas específicos; planejamento; orçamento; estudos para avaliar o impacto de intervenções específicas" (Crozara, 2001).

\section{EXPERIÊNCIAS BRASILEIRAS}

No Brasil, ainda existem poucos estudos sobre a utilização de medicamentos, sendo a maioria estudos quantitativos e apenas alguns empregam o sistema ATC de classificação de medicamentos e a unidade de medida Dose Diária Definida.

Os primeiros trabalhos, realizados na década de 80 por Simões e Farache Filho (1988) e Haak (1989), descrevem o impacto da difusão das especialidades farmacêuticas e o perfil da população que as utilizavam.

Os estudos publicados nas décadas seguintes apresentam o perfil de utilização de medicamentos pela população e grupos de risco como idosos, gestantes e crianças (Bricks, Leone, 1996; Weiderpass et al., 1998; Mosegni et al., 1999; Mengue et al., 2001; Teixeira, Lefrève, 2001; Fonseca, Fonseca, Bergsten-Mendes, 2002; Cunha, Zorzatto, Castro, 2002; Carvalho et al., 2003; Coelho Filho, 2004; Bertoldi et al., 2004; Berquó et al., 2004), da automedicação (Arrais et al., 1997; Vilarino et al., 1998; Loyola Filho et al., 2002); das prescrições de medicamentos realizada por médicos e dentistas para população ou para grupos específicos (Castilho, Paixão, Perini, 1999).
Os estudos mais recentes utilizam o Sistema ATC e expressam os resultados em Dose Diária Definida, em gastos para a instituição e/ou avaliam o impacto de intervenções educativas (Passianotto et al., 1998; Queiroz, 2000; Crozara, 2001; Ribeiro, 2002; Castro et al., 2002).

\section{Perspectivas}

Com a conscientização de que os estudos de utilização de medicamentos são imprescindíveis para a detecção, análise e solução dos problemas advindos da utilização inadequada dos medicamentos, reforça-se a tendência de que cresça o número desses estudos e das instituições que apóiem sua realização, dando-lhes condições de serem realizados com maior fidedignidade dos dados - como prescrição eletrônica, melhoria da qualidade dos prontuários, tanto em sua organização como na melhor descrição da evolução clínica.

Com recursos de informatização e um banco de dados com informações sobre os critérios do uso adequado dos medicamentos, é possível realizar estudos prospectivos e, por meio da comunicação entre médicos e farmacêuticos, corrigir possíveis erros de prescrição, logo que ocorrerem, garantindo melhor assistência aos pacientes.

Nos Estados Unidos, a partir da década de 1990, surgiram empresas de gerenciamento de medicamentos Pharmacy Benefits Management (PBM) - que se baseiam nos estudos de utilização e na farmacoeconomia para otimizar os gastos com medicamentos.

No Brasil, essas empresas também estão sendo implantadas, prestando serviços, atualmente, para redes de drogarias visando ao melhor gerenciamento de estoques. Entretanto, há perspectivas de aplicação dos Estudos de Utilização de Medicamentos na gestão pública de recursos destinados à saúde, com ênfase no consumo de medicamentos.

\section{ABSTRACT}

\section{The importance and the history of studies on medicine use}

There is a worldwide concern about rational drug use and medical treatment approach. In developing countries, the 
rational drug use represents an alternative that allows cost reduction without loosing quality in treatment. Although appropriate use of drugs may be indeed of vital importance, drugs represent a double edged word due to their potential adverse effects. The drug utilization studies are tools for detection of possible abuses on its use or the occurrence of adverse events, preventing unappropriate use and unnecessary expenses (pharmacoepidemiology and pharmacoeconomy). However, a few resources are intended for monitoring rational drug use. Moreover, there are limited data on the use of therapeutic agents in hospitals. This research confirmed the importance of these studies, especially in hospitals, to cost reduction and more secure drug use. The observation of deviations, inefficacy and adverse events on the drug utilization makes possible the development of government political and of education interventions, with the aim of rational drug utilization.

UNITERMS: Drug utilization. Pharmacoeconomy. Pharmacoepidemiology.

\section{REFERÊNCIAS BIBLIOGRÁFICAS}

ARAUJO, R.Q. Antibiótico-profilaxia em cirurgias ortopédicas: resultado da implantação de um protocolo. São Paulo, 2000. 62p. [Dissertação de Mestrado Faculdade de Ciências Médicas - Universidade Estadual de Campinas].

ARRAIS, P.S.D.; COELHO, H.L.L.; BATISTA, M.C.D.S; CARVALHO, M.L.; RIGHI, R.E.; ARNAU, J.M. Perfil da automedicação no Brasil. Rev. Saúde Pública, São Paulo, v.31, n.1, p.71-77, 1997.

BARROS, J.A.C. Propaganda de medicamentos: atentado á saúde? São Paulo: Hucitec-Sobravime, 1995. 222p.

BERMUDEZ, J.A.Z.; BONFIM, J.R.A. Medicamentos na reforma do setor saúde. São Paulo: Sobravime; 1999. $239 \mathrm{p}$.

BERQUÓ, L.S.; BARROS, A.J.D.; LIMA, R.C.; BERTOLDI, A.D. Utilização de medicamentos para tratamento de infecções respiratórias na comunidade. Rev. Saúde Pública, São Paulo, v.38, n.3, p.358-364, 2004.

BERTOLDI, A.D.; BARROS, A.J.D.; HALLAL, P.C.; LIMA, R.C. Utilização de medicamentos em adultos: prevalência e determinantes individuais. Rev. Saúde Pública, São Paulo, v.38, n.2, p.228-238, 2004.
BRASIL. Decreto n. 1018, de 01 de outubro de 1996 Regulamenta a Lei $\mathrm{n}^{\circ}$ 9.294, de 15 de julho de 1996, que dispõe sobre as restrições ao uso e à propaganda de produtos fumígenos, bebidas alcoólicas, medicamentos, terapias e defensivos agrícolas, nos termos do $\S 4^{\circ}$ do art. 220 da Constituição. Diário Oficial da União; Brasília, 02 out 1996. Disponível em: http://www.anvisa.gov.br/legis/decretos/ 2018_96.htm Acesso em: 10 ago 2005.

BRASIL. Lei n. 6360, de 23 de setembro de 1976. Dispõe sobre a vigilância sanitária a que ficam sujeitos os medicamentos, as drogas, os insumos farmacêuticos e correlatos, cosméticos, saneantes e outros produtos, e dá outras providências. Diário Oficial da União, Brasília, 24 set. 1976. Disponível em: http://www.anvisa.gov.br/ legis/leis/6360_76.htm. Acesso em: 10 ago 2005.

BRASIL. Lei n.10167, de 27 de dezembro de 2000. Altera dispositivos da Lei $n^{\circ} 9.294$, de 15 de julhode 1996, que dispõe sobre as restrições ao uso e à propaganda de produtos fumígenos, bebidas alcoólicas, medicamentos, terapias e defensivos agrícolas. Diário Oficial da União; Brasília, 28 dez 2000. Disponívelem:http:/www.anvisa.gov.br/legis/leis/ 10167_00.htm Acesso em: 10 ago 2005.

BRASIL. Portaria n. 507, de 23 de abril de 1999. Publica a revisão da Relação nacional de Medicamentos Essenciais - RENAME, que acompanha esta Portaria. Diário Oficial da União, Brasília, 04 maio 1999. Disponível em: http://e-legis.bvs.br/leisref/public/search.php Acesso em: 10 ago 2005.

BRASIL. Resolução RDC n.102, de 30 de novembro de 2000. Aprova o Regulamento sobre propagandas, mensagens publicitarias e promocionais e outras práticas cujo objeto seja adivulgação, promoção ou comercialização demedicamentos de produção nacional ou importados, quaisquer que sejam as formase meios de suaveiculação, incluindo as transmitidas no decorrer da programação normal das emissoras de rádio e televisão. Diário Oficial da União, Brasília, 01 dez 2000. Disponível em: http://www.anvisa.gov.br/legis/resol/ 102_03.htm Acesso em: 10 ago 2005.

BRICKS, L.F.; LEONE, C. Utilização de medicamentos por crianças atendidas em creches. Rev. Saúde Pública, São Paulo,v.30, n.6, p.527-535, 1996.

CAPELLÀ, D. Descriptive tools and analysis. In: CASTRO, C.GS.O., Coord. Estudos de utilização de medicamentos: noções básicas. Rio de Janeiro: Fiocruz, 2000. 90p. 
CARVALHO, P.R.A.; CARVALHO, C.G.; ALIEVI, P.T.; MARTINBIANCHO, J.; TROTTA, E.A. Identificação de medicamentos "não apropriados para crianças" em prescrições de unidade de tratamento intensivo pediátrica. J. Ped., São Paulo, v.79, n.5, p.397-402, 2003.

CASTILHO, L.S.; PAIXÃO, H.H.; PERINI, E. Prescrição de medicamentos de uso sistêmico por cirurgiõesdentistas, clínicos gerais. Rev. Saúde Pública, São Paulo, v.33, n.3, p.287-294, 1999.

CASTRO, M.S.; PILGER, D.; FERREIRA, M.B.C.; KOPITTKE, L. Tendências na utilização de antimicrobianos em um hospital universitário, 1990-1996. Rev. Saúde Pública, São Paulo, v.36, n.5, p.553-558, 2002.

CASTRO, C.G.S.O., coord. Estudos de utilização de medicamentos: noções básicas. Rio de Janeiro: Fiocruz, 2000. 90 p.

COELHO FILHO, J.M.; MARCOPITO, L.F.; CASTELO, A. Perfil de utilização de medicamentos por idosos em área urbana do nordeste do Brasil. Rev. Saúde Pública, São Paulo, v.38, n.4, p.557-564, 2004.

CROZARA, M.A. Estudo do consumo de medicamentos em hospital particular. São Paulo, 2001. 133 p. [Dissertação de Mestrado - Faculdade de Ciências Farmacêuticas - Universidade de São Paulo].

CUNHA, M.C.N.; ZORZATTO, J.R.; CASTRO, L.L.C. Avaliação do uso de medicamentos na Rede Pública Municipal de Saúde de Campo Grande/ MS. Rev. Bras. Ciênc. Farm., São Paulo, v.38, n.2, p.215-227, 2002.

FEBRAFARMA. Sufoco Geral. Rev Indústria Farmacêutica. v.jan/fev, n.4, 2004. Disponível em: http://www. febrafarma.org.br/areas.php?area $=$ pu $\&$ secao $=$ $18 \&$ modulo $=$ materias. Acesso em: 10 ago 2005.

FONSECA, M.R.C.C.; FONSECA, E.; BERGSTENMENDES, G. Prevalência do uso de medicamentos na gravidez: uma abordagem farmacoepidemiológica. Rev. Saúde Pública, São Paulo, v.36, n.2, p.205-212, 2002.

HAAK, H. Padrões de consumo de medicamentos em dois povoados da Bahia (Brasil). Rev. Saúde Pública, São Paulo, v.23, n.2, p.143-151, 1989.
HENSLEY, S. Prescriptions costs become harder to swallow. Providers and payers get a big dose of reality with explosive spending and patient demand for new drugs. Mod. Health., Chicago, v.29, n.23, p. 30-34, 1999.

KLAASSEN, C.D. Princípios de toxicologia. In: GILMAN,A.G. et al. Goodman e Gilman: as bases farmacológicas da terapêutica. 7. ed. Rio de Janeiro: Guanabara Koogan, 1985. p.1045-1052. Apud: MARTINS, D. I. Farmacovigilância e Reações adversas aos medicamentos (RAMs). Mundo Saúde, São Paulo, v.24, n.2, p.106-109, 2000.

LAPORTE, J.R.; TOGNONI, G.; ROSENFELD, S. Epidemiologia do medicamento: princípios gerais. São Paulo: HUCITEC-ABRASCO, 1989. 293p.

LENZ, W. Thalidomide: facts and inferences. In: LAPORTE, J.R.; TOGNONI, G.; ROSENFELD, S. Epidemiologia do medicamento: princípios gerais. São Paulo: HUCITEC-ABRASCO, 1989. p.43-55.

LIEBER, N.S.R. Política de medicamentos. Mundo Saúde, São Paulo, v. 24, n.1, p.45-50, 2000.

LOYOLA, A.I.F.; UCHOA, E.; GUERRA, H.L.; FIRMO, J.O.A.; LIMA-COSTA, M.F. Prevalência e fatores associados a automedicação: resultados do projeto Bambuí. Rev. Saúde Pública, São Paulo, v.36, n.1, p.5562, 2002 .

LUIZA, V.L.; CASTRO, C.G.S.O.; NUNES, J.M. Aquisição de medicamentos no setor público: o binômio qualidade - custo. Cad. Saúde Pública, Rio de Janeiro, v.15, n.4, p.769-776, 1999.

LUNDE, P.K.M. Drug and product selection - in essential part of the therapeutic benefit/risk ratio strategy? Apud: LAPORTE, J.R.; TOGNONI, G.; ROSENFELD, S. Epidemiologia do medicamento: princípios gerais. São Paulo: HUCITEC-ABRASCO, 1989. 293p.

MENGUE, S.S.; SCHENKEL, E.P.; DUNCAN, B.B.; SCHMIDT, M.I. Uso de medicamentos por gestantes em seis cidades brasileiras. Rev. Saúde Pública, São Paulo, v.35, n.5, p.415-420, 2001.

MOSEGNI, G.B.G.; ROZENFELD, S.; VERAS, R.P.; VIANNA, C.M.M. Avaliação da qualidade do uso de medicamentos em idosos. Rev. Saúde Pública, São Paulo, v.33, n.5, p.437-444, 1999. 
MOTA, D.M.; MARQUES, R.F.O.M.; FERNANDES, M.E.P. A Farmácia comunitária, a automedicação e o farmacêutico: projeções para o século XXI. Mundo Saúde, São Paulo, v.24, n.2, p. 98-105, 2000.

NASCIMENTO, M.C. A centralidade do medicamento na terapêutica contemporânea. Rio de Janeiro, 2002. 138 p. [Tese de Doutorado em Saúde Coletiva. Instituto de Medicina Social - Universidade do Estado do Rio de Janeiro].

NASCIMENTO, M.C. Medicamentos: ameaça ou apoio à saúde?: vantagens e perigos do uso de produtos da indústria farmacêutica mais consumidos no Brasil: vitaminas, analgésicos, antibióticos e psicotrópicos. Rio de Janeiro: Vieira e Lent, 2003, 200p.

NASCIMENTO, A. Ao persistirem os sintomas, o medico deverá ser consultado: Isto é regulação? São Paulo: Sociedade Brasileira de Vigilância de Medicamentos, 2005. 152p.

NELSON, B. IMS HEALTH Forecasts 7.8 percent Annual Growth in Key Latin America Pharmaceutical Markets through 2005.Disponível em: http://www.imshealth.com/ims/ portal/front/articleC/0,2777,6266_3665_1003659,00.html. Acesso em: 10 ago 2005.

PASSIANOTTO, M.M.; VILA, R.R.I.S.M.; STORPIRTIS, S.; JÚNIOR, A.F. Estudo de utilização de antimicrobianos de uso restrito no Hospital Universitário da Universidade de São Paulo empregando doses diárias definidas. Rev. Med. HU-USP, São Paulo, v.8, n.1, p.5-12, 1998.

PEPE, V.L.E.; CASTRO, C.G.S.O. A interação entre prescritores, dispensadores e pacientes: informação compartilhada como possível benefício terapêutico. $\mathrm{Cad}$. Saúde Pública, Rio de Janeiro, v.16, n.3, p.815-822, 2000.

PEREIRA, I.M.T.B.; PENTEADO, R.Z.; MARCELO, V.C. Promoção da saúde e educação em saúde: uma parceria saudável. Mundo Saúde, São Paulo, v.24, n.1, p.39-43, 2000.

REMÉDIOS: a solução é simples. O Estado de São Paulo, São Paulo, 20 jul. 1999. Caderno A, p.3

RIBEIRO, E. Avaliação do uso de um instrumento educativo para solicitação de albumina humana em um hospital universitário de São Paulo. São Paulo, 2002. 186p. [Tese de Doutorado. Faculdade de Ciências Farmacêuticas. Universidade de São Paulo].
RUIZ, I. Estudios de utilización de medicamentos. Apud: CASTRO, C.G.S.O. de. Estudos de utilização de medicamentos - noções básicas. Rio de Janeiro: Fiocruz, 2000. In: ARANCÍBIA, A.; CID, E.; DOMECQ, C.; PAULOS, C.; PEZZANI, M.; PINILLA, E.; ROJAS, M.; RUIZ, I.; VARGAS, M., org. Fundamentos de Farmácia Clínica. Santiago: Piade-Universidade de Chile, 1993.

SANTEL, J.P. Projecting future drug expenditures. Am. J. Health-Syst. Pharm., Bethesda, v.15, n.57 (2), p.129-38, 2000.

SANTI, V. de. Medicamentos: verso e reverso da propaganda. Ponta Grossa: UEPG, 1999. 114p

SIMÕES, M.J.S.; FARACHE FILHO, A. Consumo de medicamentos em região do estado de São Paulo (Brasil), 1985. Rev. Saúde Pública, São Paulo, v.22, n.6, p.494-499, 1988.

SOCIEDADE BRASILEIRA DE VIGILÂNCIA DE MEDICAMENTOS; ACCIÓN INTERNACIONAL PARA LA SALUD DA AMÉRICA LATINA Y EL CARIBE. O que é uso racional de medicamentos? São Paulo: HUCITEC-ABRASCO, 2001. 128p.

TEIXEIRA，J.J.V.; LEFÈVRE，F. A prescrição medicamentosa sob a ótica do paciente idoso. Rev. Saúde Pública, São Paulo, v.35, n.2, p.207-213, 2001.

VIDOTTI, C.C.F.; HOEFLER, R.; SILVA, E.V.; BERGSTENMENDES, G. Sistema brasileiro de informação sobre medicamentos - SISMED. Cad. Saúde Pública, Rio de Janeiro, v.16, n. 4,p.1121-1126, 2000.

VILARINO, J.F.; SOARES, I.C.; SILVEIRA, C.M.; RÖDEL, A.P.P.; BORTOLI, R; LEMOS, R.R. Perfil da automedicação em município do Sul do Brasil. Rev. Saúde Pública, São Paulo, v.32, n.1, p.43-49, 1998.

WEIDERPASS, E.; BÉRIA, J.U.; BARROS, F.C.; VICTORA, C.G.; TOMASI, E.; HALPERN, R. Epidemiologia do consumo de medicamentos no primerio trimestre de vida em centro urbano do Sul do Brasil. Rev. Saúde Pública, São Paulo, v.32, n.4, p.335-344, 1998. 
WORLD HEALTH ORGANIZATION. Report of the WHO expert commitee on national drug policies Contribution to updating the WHO guidelines for developing national drug policies, WHO - Oslo, 1988. Apud: SOCIEDADE BRASILEIRA DE VIGILÂNCIA DE MEDICAMENTOS; ACCIÓN INTERNACIONAL PARA LA SALUD DA AMÉRICA LATINA Y EL CARIBE. O que é uso racional de medicamentos? São Paulo: HUCITECABRASCO; 2001. 128p.

WORLD HEALTH ORGANIZATION. The state of world health: life expectancy, health expectancy, 1997. Disponível em: www.who.int/whr. Acesso em: 8 maio 2003.
WORLD HEALTH ORGANIZATION/ Who Collaborating Centre For Drug Statistics Methodology. Guidelines for DDD. Oslo: WHO - Oslo, 1993a. Apud: CASTRO, C.G.S.O., coord. Estudos de utilização de medicamentos: noções básicas. Rio de Janeiro: Fiocruz, 2000. 90p.

WORLD HEALTH ORGANIZATION. The world health report 2006 - working together for health. Disponível em:http://www.who.int/whr/2006. Acesso em: 14 de setembro de 2006.

Recebido para publicação em 23 de novembro de 2005 Aceito para publicação em 19 de novembro de 2006 